\title{
Modeling $\beta$ Virginis using seismological data
}

\author{
P. Eggenberger and F. Carrier
}

Observatoire de Genève, 51 chemin des Maillettes, 1290 Sauverny, Switzerland
e-mail: Patrick.Eggenberger@obs. unige.ch

Received 15 February 2005 / Accepted 28 October 2005

\section{ABSTRACT}

This paper presents the modeling of the F9 V star $\beta$ Virginis based on seismological measurements. Using the Geneva evolution code including rotation and atomic diffusion, we find that two distinct solutions reproduce all existing asteroseismic and non-asteroseismic observational constraints well: a main-sequence model with a mass of $1.28 \pm 0.03 M_{\odot}$ and an age $t=3.24 \pm 0.20 \mathrm{Gyr}$, or a model in the post-main sequence phase of evolution with a lower mass of $1.21 \pm 0.02 M_{\odot}$ and an age $t=4.01 \pm 0.30 \mathrm{Gyr}$. The small spacings $\delta v_{02}$ and the ratio $r_{02}$ between small and large spacings are sensitive to the differences in the structure of the central layers between these two solutions and are also sensitive to the structural changes due to the rotational mixing. They can therefore be used to unambiguously determine the evolutionary state of $\beta$ Vir and to study the effects of rotation on the inner structure of the star. Unfortunately, existing asteroseismic data do not enable such precise determination. We also show that the scatter in frequencies introduced by the rotational splittings can account for the larger dispersion of the observed large spacings for the non-radial modes than for the radial modes.

Key words. stars: individual: $\beta$ Virginis - stars: evolution - stars: oscillations

\section{Introduction}

The solar five-minute oscillations have led to a wealth of information about the internal structure of the Sun. These results stimulated various attempts to detect a similar signal on other solar-like stars by photometric or equivalent width measurements. In past years, the stabilized spectrographs developed for an extra-solar planet search have finally achieved the accuracy needed to make these detections. While solar-like oscillations have been detected for a handful of solar-type stars, individual p-mode frequencies have only been identified for a few of these stars: $\alpha$ Cen A (Bouchy \& Carrier 2002; Bedding et al. 2004), $\alpha$ Cen B (Carrier \& Bourban 2003; Kjeldsen et al. 2005), Procyon A (Martić et al. 2004a; Eggenberger et al. 2004a), $\eta$ Bootis (Kjeldsen et al. 2003; Carrier et al. 2005a), and $\mu$ Arae (Bouchy et al. 2005). Individual frequencies have also been identified in the giant star $\xi$ Hydrae (Frandsen et al. 2002).

Based on these asteroseismic data, numerous theoretical analyses have been performed in order to determine precise global stellar parameters (see for instance Eggenberger et al. $2004 \mathrm{~b}$ for the $\alpha$ Cen system) and to try to test the inclusion of overshooting or rotation in the models (see Di Mauro et al. 2003 for $\eta$ Boo or Eggenberger et al. 2005a for Procyon).

Recently, Carrier et al. (2005b) detected solar-like oscillations on the F9 $\mathrm{V}$ star $\beta$ Virginis (HD 102870) and reported identification of several individual frequencies. Martić et al. (2004b) also detected solar-like oscillations on $\beta$ Virginis, but no individual frequencies were identified. The aim of the present paper is to investigate which additional constraints are brought by these seismological data. We will thus try to determine the model of $\beta$ Virginis that best reproduces all the observational constraints using the Geneva evolution code, which includes a comprehensive treatment of shellular rotation and atomic diffusion.

The observational constraints available for $\beta$ Virginis are summarized in Sect. 2, while the computational method is described in Sect. 3. The results are presented in Sect. 4 and the effects of rotation are discussed in Sect. 5. The conclusion is given in Sect. 6.

\section{Observational constraints}

\subsection{Effective temperature and chemical composition}

From the different spectroscopic measurements reported in the literature for $\beta$ Vir (Edvardsson et al. 1993; Buzzoni et al. 2001; Gray et al. 2001; Le Borgne et al. 2003; Taylor et al. 2003; Morel \& Micela 2004; Allende Prieto et al. 2004; Lambert et al. 2004), we adopt an effective temperature $T_{\text {eff }}=6130 \pm 50 \mathrm{~K}$ and a metallicity $[\mathrm{Fe} / \mathrm{H}]=0.14 \pm 0.05$.

\subsection{Luminosity}

Combining the magnitude $V=3.597 \pm 0.004$ (Burki et al. 2005), the Hipparcos parallax $\Pi=91.74 \pm 0.77$ mas, the solar absolute bolometric magnitude $M_{\mathrm{bol}, \odot}=4.746$ (Lejeune et al. 1998) and the bolometric corrections from Flower's (1996) 
Table 1. Non-asteroseismic observational constraints for $\beta$ Vir.

\begin{tabular}{cc}
\hline \hline$\Pi[\mathrm{mas}]$ & $91.74 \pm 0.77$ \\
$V[\mathrm{mag}]$ & $3.597 \pm 0.004$ \\
$L / L_{\odot}$ & $3.51 \pm 0.10$ \\
$T_{\mathrm{eff}}[\mathrm{K}]$ & $6130 \pm 50$ \\
{$[\mathrm{Fe} / \mathrm{H}]$} & $0.14 \pm 0.05$ \\
$V \sin i\left[\mathrm{~km} \mathrm{~s}^{-1}\right]$ & $\sim 4.3$ \\
\hline
\end{tabular}

$B C=-0.028 \pm 0.006$ mag determined from the effective temperature, we find a luminosity $L=3.51 \pm 0.10 L_{\odot}$.

\subsection{Rotational velocity}

We use the observed surface velocity of $\beta$ Vir to constrain the rotational velocity of our models. Glebocki \& Stawikowski (2000) have determined a velocity $V \sin i \cong 4.3 \mathrm{~km} \mathrm{~s}^{-1}$. Since the value of the angle $i$ is unknown, we assume that it is close to $90^{\circ}$. Thus our models of $\beta$ Vir have to reproduce a surface velocity of about $4.3 \mathrm{~km} \mathrm{~s}^{-1}$.

All non-asteroseismic observational constraints are listed in Table 1.

\subsection{Asteroseismic constraints}

Solar-like oscillations in $\beta$ Vir have been detected by Carrier et al. (2005b) with the CORALIE echelle spectrograph. Thirty-one oscillation frequencies have been identified in the power spectrum between 0.7 and $2.4 \mathrm{mHz}$ with amplitudes in the range 23 to $46 \mathrm{~cm} \mathrm{~s}^{-1}$. By a least square fit of the asymptotic relation with the identified frequencies, they determined a mean large and small separation of $\Delta v=72.1 \mu \mathrm{Hz}$ and $\delta v_{02}=6.3 \mu \mathrm{Hz}$, respectively. The precision of the frequency determination is estimated by conducting Monte Carlo simulations in which time series computed for various values of the damping time and re-excitation rates are analyzed. A velocity time series for detected oscillation modes is built by using the observational time sampling and the observational noise on radial velocities. The amplitude spectrum of this series is then calculated and the differences between the simulated peaks, and the actual frequencies are determined. The whole procedure is repeated 1000 times for each set of parameters to ensure the stability of the results. These simulations lead to an estimation of the error on individual frequency between 0.8 and $1.4 \mu \mathrm{Hz}$, mainly depending on the damping time. This result is very close to the frequency resolution of the time series $(1.14 \mu \mathrm{Hz})$. In the following, we thus adopt an error on individual frequencies estimated as the frequency resolution of the time series.

\section{Stellar models}

\subsection{Code description}

\subsubsection{General input physics}

The stellar evolution code used for these computations is the Geneva code that includes shellular rotation (Meynet \& Maeder 2000). We use the OPAL opacities, the NACRE nuclear reaction rates (Angulo et al. 1999), and the standard mixinglength formalism for convection. We do not include an additional convective penetration from the convective core into the surrounding stable layers, since the inclusion of shellular rotation results in an increase of the convective core (see Fig. 11 of Carrier et al. 2005a).

\subsubsection{Rotation}

We follow the evolution of the rotation profile from the zero age main sequence on, assuming initial solid body rotation. The braking law of Kawaler (1988) is used in order to reproduce the magnetic braking undergone by low mass stars when arriving on the main sequence. Two parameters enter this braking law: the saturation velocity $\Omega_{\text {sat }}$ and the braking constant $K$. Following Bouvier et al. (1997), $\Omega_{\text {sat }}$ is fixed to $14 \Omega_{\odot}$ and the braking constant $K$ is calibrated on the Sun.

\subsubsection{Meridional circulation}

The velocity of the meridional circulation in the case of shellular rotation was initially derived by Zahn (1992). The effects of the vertical $\mu$-gradient $\nabla_{\mu}$ and of the horizontal turbulence on meridional circulation were then taken into account by Maeder \& Zahn (1998). They find

$$
\begin{aligned}
U(r)= & \frac{P}{\rho g C_{P} T\left[\nabla_{\mathrm{ad}}-\nabla+(\varphi / \delta) \nabla_{\mu}\right]} \\
& \times\left\{\frac{L}{M}\left(E_{\Omega}+E_{\mu}\right)\right\},
\end{aligned}
$$

where $P$ is the pressure and $C_{P}$ the specific heat. The $E_{\Omega}$ and $E_{\mu}$ terms depend on the $\Omega$ - and $\mu$-distributions, respectively, up to the third order derivatives and on various thermodynamic quantities (see Maeder \& Zahn 1998 for more details).

\subsubsection{Shear turbulence}

The diffusion by shear instabilities is expressed by a coefficient $D_{\text {shear: }}$ :

$$
\begin{aligned}
D_{\text {shear }}= & \frac{4\left(K+D_{\mathrm{h}}\right)}{\left[\frac{\varphi}{\delta} \nabla_{\mu}\left(1+\frac{K}{D_{\mathrm{h}}}\right)+\left(\nabla_{\mathrm{ad}}-\nabla_{\mathrm{rad}}\right)\right]} \\
& \times \frac{H_{\mathrm{p}}}{g \delta}\left[\frac{\alpha}{4}\left(f \Omega \frac{\mathrm{d} \ln \Omega}{\mathrm{d} \ln r}\right)^{2}-\left(\nabla^{\prime}-\nabla\right)\right],
\end{aligned}
$$

where $f$ is a numerical factor equal to $0.8836, K$ the thermal diffusivity, and $\left(\nabla^{\prime}-\nabla\right)$ expresses the difference between the internal nonadiabatic gradient and the local gradient (Maeder \& Meynet 2001).

\subsubsection{Horizontal turbulence}

We used the new horizontal turbulence prescription of Maeder (2003) that expresses the balance between the energy dissipated by the horizontal turbulence and the excess of energy present 
in the differential rotation on an equipotential that can be dissipated in a dynamical time:

$D_{\mathrm{h}}=\operatorname{Ar}(r \Omega(r) V[2 V-\alpha U])^{\frac{1}{3}}$,

with

$A=\left(\frac{3}{400 n \pi}\right)^{\frac{1}{3}}$,

where $U(r)$ is the vertical component of the meridional circulation velocity, $V(r)$ the horizontal component, and $\alpha=\frac{1}{2} \frac{\mathrm{d} \ln r^{2} \Omega}{\mathrm{d} \ln r}$.

\subsubsection{Transport of chemicals}

The vertical transport of chemicals through the combined action of vertical advection and strong horizontal diffusion can be described as a pure diffusive process (Chaboyer \& Zahn 1992). Indeed, the advective transport can be replaced by a diffusive term, with an effective diffusion coefficient

$D_{\text {eff }}=\frac{|r U(r)|^{2}}{30 D_{\mathrm{h}}}$,

where $D_{\mathrm{h}}$ is the coefficient of horizontal diffusion (see Eq. (3)).

Atomic diffusion driven by gravitational settling and thermal gradients is included using the prescription by Paquette et al. (1986). The evolution of an element concentration per unit mass $c_{i}$ then follows:

$\rho \frac{\mathrm{d} c_{i}}{\mathrm{~d} t}=\dot{c}_{i}+\frac{1}{r^{2}} \frac{\partial}{\partial r}\left[r^{2} \rho\left\{U_{\mathrm{diff}} c_{i}+\left(D_{\text {eff }}+D_{\text {shear }}\right) \frac{\partial c_{i}}{\partial r}\right\}\right]$

with $U_{\text {diff }}$ the microscopic diffusion velocity and $\dot{c}_{i}$ the variations of chemical composition due to nuclear reactions.

\subsection{Computational method}

The characteristics of a stellar model that includes the effects of rotation depend on six modeling parameters: the mass $M$ of the star, its age (hereafter $t$ ), the mixing-length parameter $\alpha \equiv l / H_{\mathrm{p}}$ for convection, the initial surface velocity $V_{\mathrm{i}}$, and two parameters describing the initial chemical composition of the star. For these two parameters, we choose the initial hydrogen abundance $X_{\mathrm{i}}$ and the initial ratio between the mass fraction of heavy elements and hydrogen $(Z / X)_{\mathrm{i}}$. This ratio is directly related to the abundance ratio $[\mathrm{Fe} / \mathrm{H}]$ assuming that $\log (Z / X) \cong[\mathrm{Fe} / \mathrm{H}]+\log (Z / X)_{\odot}$; we use the solar value $(Z / X)_{\odot}=0.0230$ given by Grevesse \& Sauval (1998).

Contrary to our previous seismic calibrations of binary stars like $\alpha$ Centauri and Procyon, we decided to limit the parameter space of the present analysis by fixing the mixing-length parameter to its solar calibrated value $\left(\alpha_{\odot}=1.75\right)$ and the hydrogen abundance to $X=0.7$. This simply results from the limited number of non-asteroseismic observational constraints available for a star like $\beta$ Virginis, which does not belong to a binary system. Although this arbitrarily limits our analysis to a small subset of possible solutions, we think that it is the appropriate way to proceed for a first modeling of an isolated star in order to investigate which constraints are brought by the observed p-mode frequencies. A more thorough exploration of

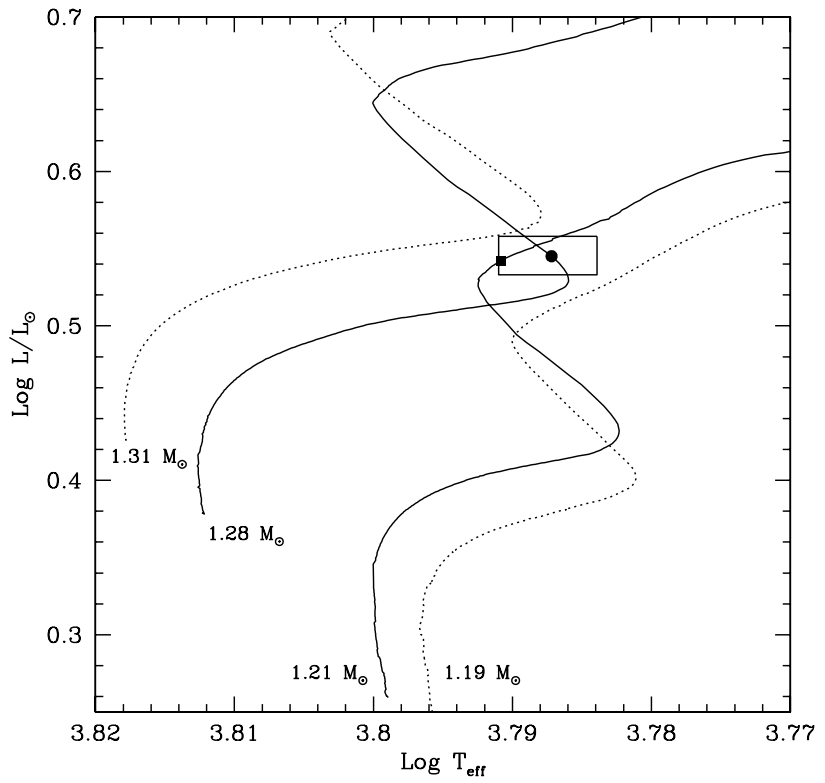

Fig. 1. Evolutionary tracks in the HR diagram for the two models of $\beta$ Vir. The dot and the square indicate the location of the M 1 and M 2 models, respectively. The error box in continuous line indicates the observational constraints of $L$ and $T_{\text {eff. }}$. The evolutionary tracks of a $1.19 M_{\odot}$ and $1.31 M_{\odot}$ model are also plotted (dotted lines); this shows that models with masses that are lower than $1.19 M_{\odot}$ or larger than $1.31 M_{\odot}$ are not able to reproduce the non-asteroseismic constraints.

the parameter space, leading to the independent determination of the mixing-length parameter and the hydrogen abundance, will be possible when additional and more accurate seismic and non-asteroseismic observables (e.g. interferometric radius) are available for $\beta$ Virginis.

We then construct a grid of models with position in the HR diagram in agreement with the observational values of the luminosity and effective temperature (see Fig. 1). Note that the initial ratio between the mass fraction of heavy elements and hydrogen $(Z / X)_{\mathrm{i}}$ is directly constrained by the observed surface metallicity $[\mathrm{Fe} / \mathrm{H}]$, while the initial velocity $V_{\mathrm{i}}$ is directly constrained by the observed rotational velocity. For each stellar model of this grid, low- $\ell$ p-mode frequencies are calculated using the Aarhus adiabatic pulsations package written by J. Christensen-Dalsgaard (1997). Following the observations, only modes $\ell \leq 2$ with frequencies between 0.7 and $2.4 \mathrm{mHz}$ are computed. To determine the set of modeling parameters $\left(M, t, V_{\mathrm{i}},(Z / X)_{\mathrm{i}}\right)$ that leads to the best agreement with the observational constraints, two functionals are defined: $\chi_{\text {clas }}^{2}$ and $\chi_{\text {astero }}^{2}$. The $\chi_{\text {clas }}^{2}$ functional only uses the "classical" (i.e. non-asteroseismic) observables, and is defined as follows

$\chi_{\text {clas }}^{2} \equiv \frac{1}{3} \sum_{i=1}^{3}\left(\frac{C_{i}^{\text {theo }}-C_{i}^{\text {obs }}}{\sigma C_{i}^{\text {obs }}}\right)^{2}$,

where the vector $\mathbf{C}$ contains the following observables for one star:

$C \equiv\left(L / L_{\odot}, T_{\mathrm{eff}},[\mathrm{Fe} / \mathrm{H}]\right)$.

The vector $\boldsymbol{C}^{\text {theo }}$ contains the theoretical values of these observables for the model to be tested, while the values of $\boldsymbol{C}^{\text {obs }}$ 
are those listed in Table 1 . The vector $\sigma \mathbf{C}$ contains the errors on these observations. Note that the observed rotational velocity is not included in this minimization, because of its large uncertainty resulting from the unknown inclination angle $i$. The $\chi_{\text {astero }}^{2}$ functional compares individual theoretical frequencies to the observed ones. The exact values of the computed p-mode frequencies depend on the details of the star's atmosphere, where the pulsation is non-adiabatic and where turbulent and radiative losses are significant. The discrepancy between observed and theoretical solar p-mode frequencies calculated from standard models is indeed caused primarily by the approximations made in modeling the surface layers (Ulrich \& Rhodes 1983; Christensen-Dalsgaard et al. 1988; Guenther 1994; Li et al. 2002). The effects of poor modeling of the external layers on computed p-mode frequencies can be taken into account by using the discrepancies between observed and theoretical solar frequencies (see Guenther \& Brown 2004). However, we do not know whether this estimation is reliable for stars with different surface gravity, chemical composition, and age. Thus, we prefer to simply apply a constant shift to correct theoretical frequencies for surface effects. We are well aware that this simple correction may seem doubtful, given the strong frequency dependence of the solar near-surface effects, but we think that this is the least biased procedure possible. We therefore define the mean value of the differences between the theoretical and observed frequencies:

$\left\langle D_{v}\right\rangle \equiv \frac{1}{N} \sum_{i=1}^{N}\left(v_{i}^{\text {theo }}-v_{i}^{\text {obs }}\right)$,

and then define the $\chi_{\text {astero }}^{2}$ functional as

$\chi_{\text {astero }}^{2} \equiv \frac{1}{N} \sum_{i=1}^{N}\left(\frac{v_{i}^{\text {theo }}-v_{i}^{\text {obs }}-\left\langle D_{v}\right\rangle}{\sigma}\right)^{2}$,

where $\sigma=1.14 \mu \mathrm{Hz}$ is the error on the observed frequencies, estimated as the frequency resolution of the time series (see Sect. 2.4), and $N$ is the number of observed frequencies. Note that the adopted value of the error on the observed frequencies will of course change the values of the computed $\chi_{\text {astero }}^{2}$ (a larger value of $\sigma$ resulting in smaller values of $\chi_{\text {astero }}^{2}$ and vice versa) but not the result of the minimization of the $\chi_{\text {astero }}^{2}$ functional. Carrier et al. (2005b) report the identification of thirty-one individual frequencies. However, four modes are identified at high frequencies and are thus far from the other modes, which makes their identification less secure. Moreover, these frequencies are found to be larger than the typical value of the acoustical cut-off frequency of our models of $\beta \operatorname{Vir}\left(v_{\mathrm{ac}} \cong 2.1 \mathrm{mHz}\right)$. As a result, these four frequencies are not considered in our minimization, and $N$ is hence equal to 27 instead of 31 .

Determination of the best set of parameters is then based on the simultaneous minimization of the two functionals defined in Eqs. (7) and (8). Finally, the global agreement between the stellar models and all classical and asteroseismic observational constraints available for $\beta$ Vir is tested by defining the $\chi_{\text {tot }}^{2}$ functional as the sum of $\chi_{\text {clas }}^{2}$ and $\chi_{\text {astero }}^{2}$ :

$\chi_{\text {tot }}^{2} \equiv \chi_{\text {clas }}^{2}+\chi_{\text {astero }}^{2}$.
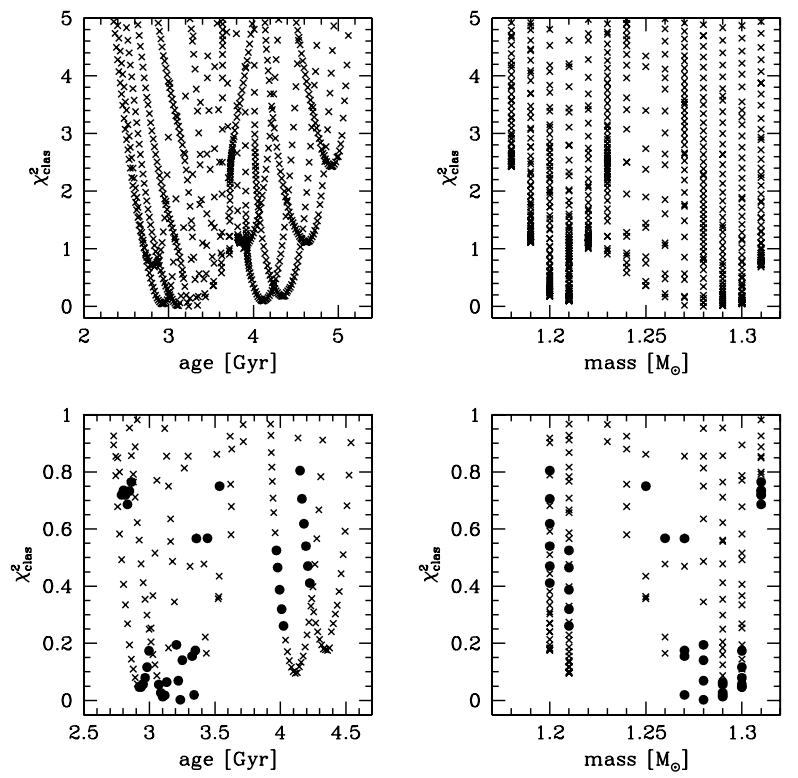

Fig. 2. Top: $\chi_{\text {clas }}^{2}$ of the models as a function of age and mass. Bottom: Zoom of the above figures. Models with $\chi_{\text {astero }}^{2}>10$ are still denoted by crosses, while models with $\chi_{\text {astero }}^{2} \leq 10$ are indicated by dots.

\section{Results}

Using the observational constraints listed in Sect. 2 with the observed frequencies of Carrier et al. (2005b), we perform the $\chi^{2}$ minimization described above.

First, only the non-asteroseismic constraints are considered. Thus the $\chi_{\text {clas }}^{2}$ minimization, which tests the consistency between observed and theoretical effective temperature, luminosity and metallicity, is applied. In this way, we find that two kind of solutions are able to reproduce the nonasteroseismic observational constraints of $\beta$ Vir: one model with a mass of about $1.21 M_{\odot}$ and an age of about $4.1 \mathrm{Gyr}$, and a more massive model of $1.28 M_{\odot}$ with a smaller age of about 3.2 Gyr. This is shown in Fig. 2, which plots the values of $\chi_{\text {clas }}^{2}$ for models matching the observed surface metallicity of $\beta$ Vir as a function of age and mass. This figure clearly shows one minimum near $1.21 M_{\odot}$ (corresponding to the minimum at $4.1 \mathrm{Gyr}$ ) and another minimum near $1.28 M_{\odot}$ (3.2 Gyr). To understand why two distinct solutions are found to fall within the observed constraints on effective temperature, luminosity and surface metallicity, we plot the evolutionary tracks in the HR diagram corresponding to the 1.21 and $1.28 M_{\odot}$ models (see Fig. 1). Figure 1 shows that the two solutions correspond in fact to two different evolutionary stages: the less massive model of $1.21 M_{\odot}$ has exhausted its hydrogen core and is therefore in the post-main sequence phase of evolution, while the $1.28 M_{\odot}$ is still on the main sequence but is very near hydrogen exhaustion. Since these two models have the same surface metallicity, the same surface velocity ( $V \cong 4 \mathrm{~km} \mathrm{~s}^{-1}$ ), and the same position in the HR diagram, non-asteroseismic observational constraints do not enable us to determine the evolutionary stage of $\beta$ Vir. Asteroseismic observations are needed to differentiate models with different internal structure located in the same region of the HR diagram. Thus, we now examine which additional 

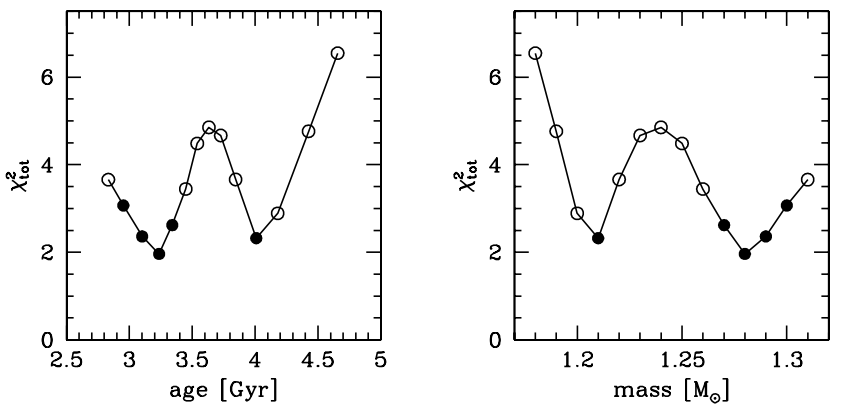

Fig. 3. Lowest values of $\chi_{\text {tot }}^{2} \equiv \chi_{\text {clas }}^{2}+\chi_{\text {astero }}^{2}$ as a function of age and mass. Dots indicate models in accordance with the observed luminosity and effective temperature, while open circles correspond to models that do not reproduce all non-asteroseismic observables.

constraints are brought by the asteroseismic measurements by performing the whole minimization described in Sect. 3 .

For this purpose, the $\chi_{\text {astero }}^{2}$ values, which directly compare individual theoretical frequencies to the observed ones, are computed. The bottom of Fig. 2 shows which models are in reasonable agreement with the observed p-mode frequencies (with $\chi_{\text {astero }}^{2} \leq 10$ ) and which models do not reproduce the asteroseismic constraints $\left(\chi_{\text {astero }}^{2}>10\right)$. We see that models still on the main-sequence, which minimize $\chi_{\text {clas }}^{2}$ for a mass and a corresponding age of about $1.28 M_{\odot}$ and $3.2 \mathrm{Gyr}$ respectively, are found to also agree well with the seismological measurements, and hence to minimize $\chi_{\text {clas }}^{2}$ and $\chi_{\text {astero }}^{2}$ at the same time. However, models in the post-main sequence phase of evolution, which minimizes $\chi_{\text {clas }}^{2}$ for a mass and a corresponding age of about $1.21 M_{\odot}$ and $4.1 \mathrm{Gyr}$, have values of $\chi_{\text {astero }}^{2}$ that are higher than ten and therefore badly reproduce the asteroseismic observables. Thus, post-main sequence models are not able to minimize the two functionals $\chi_{\text {clas }}^{2}$ and $\chi_{\text {astero }}^{2}$ at the same time as expected for a fully consistent model of $\beta$ Vir. However, the bottom of Fig. 2 shows that there are post-main sequence models that are compatible with the observed frequencies and that also have small values of $\chi_{\text {clas }}^{2}$, even if they do not minimize $\chi_{\text {clas }}^{2}$. This means that a post-main sequence model of $\beta$ Vir exists with position in the HR diagram in agreement with the observed luminosity and effective temperature, which also reproduces the observed $p$-mode frequencies well. This can be seen clearly by plotting the lowest values of the $\chi_{\text {tot }}^{2}$ functional, which tests the global agreement between the models and all observational constraints, as a function of age and mass (see Fig. 3). Figure 3 shows that there is a clear solution near $1.28 M_{\odot}$ that minimizes all $\chi^{2}$ functionals at the same time. Figure 3 also shows that the post-main sequence models near $1.21 M_{\odot}$ have low values of $\chi_{\text {tot }}^{2}$ and are therefore in good agreement with all observational constraints. At this stage of the analysis, we conclude that the additional asteroseismic measurements favor the solution of a model still on the main sequence, but do not rule out the other solution of a less massive model in the post-main sequence phase of evolution.

Thus, two different solutions are determined: a mainsequence model (denoted model M 1 in the following) and a post-main sequence model (the M 2 model). For each model, the location in the HR diagram is shown in Fig. 1 and the
Table 2. Models for $\beta$ Vir including rotation and atomic diffusion. The upper part of the table gives the non-asteroseismic observational constraints used for the calibration. The middle part of the table presents the modeling parameters with their confidence limits, while the bottom part presents the global parameters of the star.

\begin{tabular}{c|cc}
\hline \hline & Model M 1 & Model M 2 \\
\hline$L / L_{\odot}$ & \multicolumn{2}{|c}{$3.51 \pm 0.10$} \\
$T_{\text {eff }}[\mathrm{K}]$ & \multicolumn{2}{|c}{$6130 \pm 50$} \\
{$[\mathrm{Fe} / \mathrm{H}]$} & \multicolumn{2}{|c}{$0.14 \pm 0.05$} \\
$V\left[\mathrm{~km} \mathrm{~s}^{-1}\right]$ & \multicolumn{2}{|c}{$\sim 4.3$} \\
\hline$M\left[M_{\odot}\right]$ & $1.28 \pm 0.03$ & $4.21 \pm 0.02$ \\
$t[\mathrm{Gyr}]$ & $3.24 \pm 0.20$ & 0.30 \\
$V_{\mathrm{i}}\left[\mathrm{km} \mathrm{s}^{-1}\right]$ & $\sim 18$ & 0.319 \\
$(Z / X)_{\mathrm{i}}$ & $0.0340 \pm 0.0040$ & 2.003 \\
\hline$\chi_{\text {clas }}^{2}$ & 0.002 & 2.322 \\
$\chi_{\text {astero }}^{2}$ & 1.961 & 3.49 \\
$\chi_{\text {tot }}^{2}$ & 1.963 & 6177 \\
$L / L_{\odot}$ & 3.51 & 1.634 \\
$T_{\text {eff }}[\mathrm{K}]$ & 6126 & 0.14 \\
$R / R_{\odot}$ & 1.666 & 0.0317 \\
{$[\mathrm{Fe} / \mathrm{H}]$} & 0.14 & 3.9 \\
$(Z / X)$ & 0.0317 & 72.1 \\
$V\left[\mathrm{~km} \mathrm{~s}{ }^{-1}\right]$ & 4.2 & 5.5 \\
$\Delta v[\mu \mathrm{Hz}]$ & 72.1 & \\
$\delta v_{02}[\mu \mathrm{Hz}]$ & 5.4 & \multicolumn{2}{|c}{} \\
\hline
\end{tabular}

respective characteristics given in Table 2. The confidence limits of each modeling parameter are estimated as the maximum/minimum values that fit the observational constraints when the other calibration parameters are fixed to their mean values. Both models have a similar value of $\chi_{\text {astero }}^{2}$, while the M 2 model has a higher value of $\chi_{\text {clas }}^{2}$, which is mainly due to its high effective temperature (see Fig. 1).

We recall that these results have been obtained by fixing the mixing-length parameter to its solar calibrated value $\left(\alpha_{\odot}=1.75\right)$ and the hydrogen abundance to $X=0.7$, since we think that current observational data do not allow an independent determination of these quantities. We nevertheless investigated the effect of a change to these values on the determined global parameters. Increasing the mixing-length parameter $\alpha$ by $10 \%$ for a given value of the hydrogen abundance results in an increase of about 0.1 in the $\chi_{\text {clas }}^{2}$ in order to reach the same value of $\chi_{\text {astero }}^{2}$; this also decreases the mass by about $2 \%$ and increases the age by about $10 \%$. In the same way, increasing the hydrogen abundance by $10 \%$ for a fixed value of the mixinglength parameter leads to an increase of about 0.7 in the $\chi_{\text {clas }}^{2}$ in order to reach the same value of $\chi_{\text {astero }}^{2}$; this increases the mass by about $12 \%$ and decreases the age by about $6 \%$. For a given mass, an increase in the hydrogen abundance can also be compensated for an increase in the mixing-length parameter and vice versa in order to reach the same location in the HR diagram (see Fig. 3 of Eggenberger et al. 2005a).

To study the asteroseismic features of the models, we first plot the variation of the large spacing $\left(\Delta v_{n, \ell} \equiv v_{n, \ell}-v_{n-1, \ell}\right)$ 

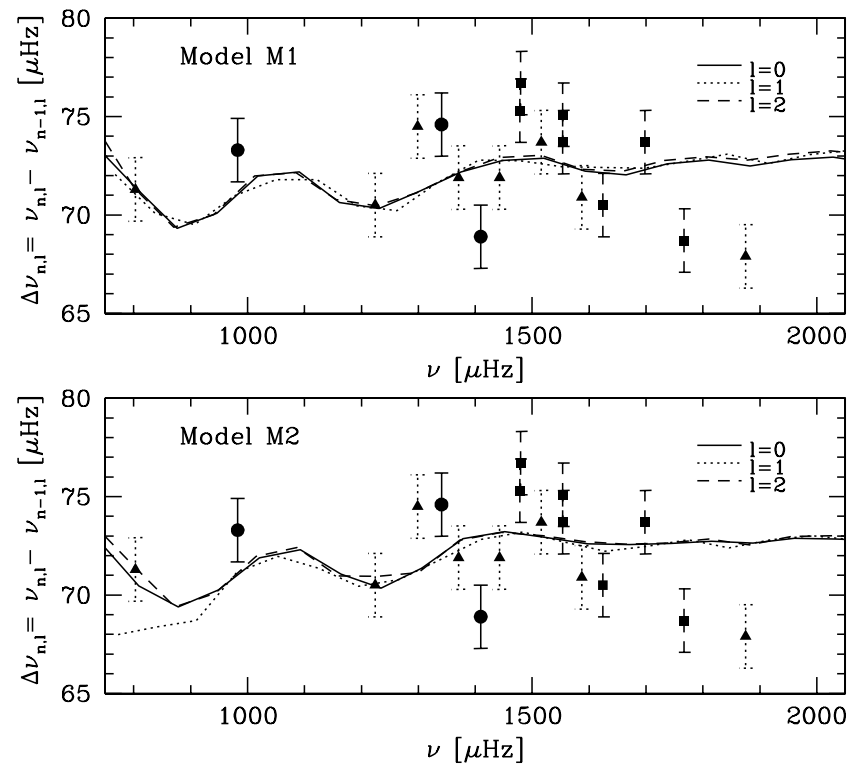

Fig. 4. Large spacings versus frequency for the M 1 and M 2 models. Dots indicate the observed values of the large spacing for radial modes, while triangles and squares correspond to the observed large spacings determined from $\ell=1$ and $\ell=2$ modes, respectively.

with frequency; the comparison between observed and theoretical large spacings for the M 1 and M 2 model is given in Fig. 4. Table 2 and Fig. 4 show that the mean large spacing of both models are in good agreement with the observed value of $72.1 \mu \mathrm{Hz}$. Note that the radius of the M 2 model is less than the one of the M 1 model in order to compensate for the decrease in mass and to reproduce the observed value of the mean large spacing. The variations in the theoretical large spacings with frequency are also very similar for both models. The only difference is that the M 2 model exhibits slightly lower values of the $\ell=1$ large spacings at low frequency than does the M 1 model. As a result, the M 1 model is able to reproduce the observed $\ell=1$ large spacing at $800 \mu \mathrm{Hz}$, whereas the theoretical value of the M 2 model is lower than the observed one. This is due to the fact that the M 2 model does not reproduce the observed $\ell=1$ mode at $732 \mu \mathrm{Hz}$ well (see Fig. 7). Finally, we note that the dispersion of the observed large spacings around the theoretical curves is greater than expected taking an uncertainty of $1.14 \mu \mathrm{Hz}$, the frequency resolution of the time series, on the frequency determination.

The small spacing $\delta v_{02} \equiv v_{n, \ell=0}-v_{n-1, \ell=2}$ is principally sensitive to the conditions in the central regions of the star. However, it also retains some sensitivity to the near-surface structure. Roxburgh \& Vorontsov (2003) introduces the use of a new asteroseismic diagnostic: the ratio of small to large spacings $r_{02} \equiv \delta v_{02} / \Delta v_{n, \ell=1}$. This ratio constitutes a better diagnostic of the central parts of a star than the small spacing, since it is essentially independent of the structure of the outer layers, and is determined solely by the interior structure (Roxburgh \& Vorontsov 2003; Otí Floranes et al. 2004).

The variation in the small spacing $\delta v_{02}$ and the ratio $r_{02}$ with frequency for the M 1 and M 2 models is given in Fig. 5.
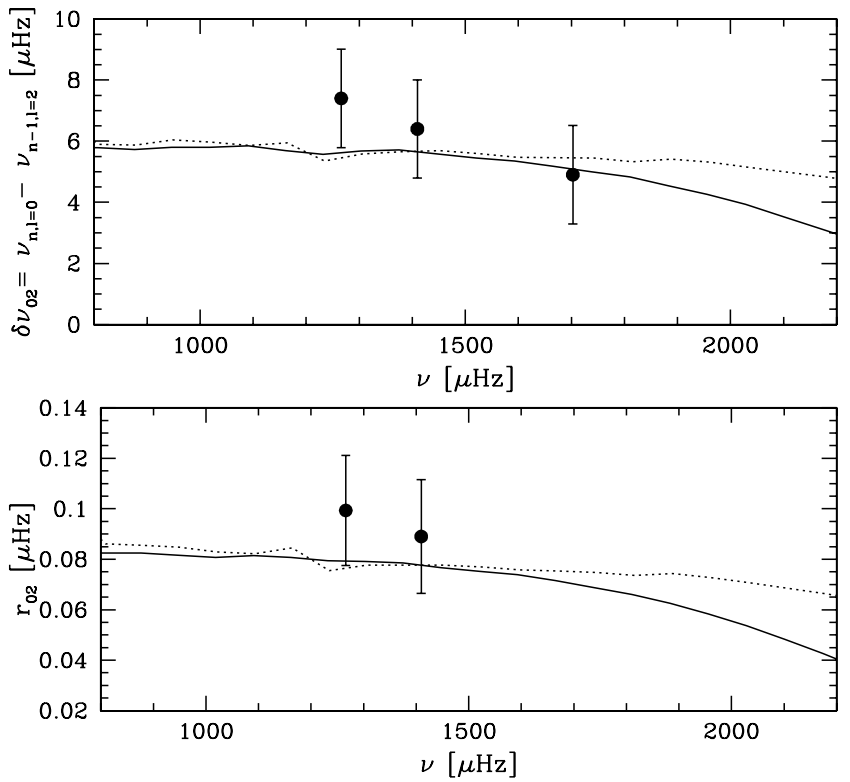

Fig. 5. Small spacing $\delta v_{02}$ and ratio of small to large separation $r_{02} \equiv$ $\delta v_{02} / \Delta v_{n, \ell=1}$ as a function of frequency. The continuous line corresponds to the M 1 model, while the dotted line corresponds to the M 2 model. Dots indicate the observed values.

The large spacing corresponding to the observed small spacing near $1700 \mu \mathrm{Hz}$ has not been observed; thus, only two observational values of the $r_{02}$ ratio are available. We see that the theoretical mean small spacing of both models (see Table 2) is smaller than the observed value of $6.3 \pm 1.4 \mu \mathrm{Hz}$. Note that the theoretical mean small spacing has been determined by only considering the three theoretical values corresponding to the observed small spacings, in order to correctly compare stellar models to observations. Figure 5 shows that the difference between the theoretical and observed mean small spacings is mainly due to the large observational value of $\delta v_{02}$ near $1270 \mu \mathrm{Hz}$, which models fail to reproduce. This is also true for the $r_{02}$ ratio. The variation in $\delta v_{02}$ and $r_{02}$ with frequency is very similar for both models at low frequency, but is found to be quite different at higher frequency. Indeed, Fig. 5 shows that the M 1 and M 2 models are characterized by approximately the same values of $\delta v_{02}$ and $r_{02}$ at frequencies lower than about $1600 \mu \mathrm{Hz}$, while at higher frequencies, the M 1 model exhibits a significantly stronger decrease in $\delta v_{02}$ and $r_{02}$ than does the M 2 model. This is directly related to the evolutionary stage of the two models. Indeed, the post-main-sequence M 2 model is more centrally condensed and therefore exhibits higher central values of pressure and density than the younger M 1 model, which is approaching hydrogen exhaustion but is still on the main-sequence. Consequently, the sound speed and hence the Lamb frequency of the two models differ in the central parts of the star. The interior reflection of the $\ell=2$ modes are then different for the two models, which results in a stronger decrease in $\delta v_{02}$ and $r_{02}$ for the M 1 model than for the M 2 model. Unfortunately, only one value for $\delta v_{02}$ and none for $r_{02}$ have been observed for a frequency higher than $1600 \mu \mathrm{Hz}$. The M 1 model agrees slightly better with this value of the small 


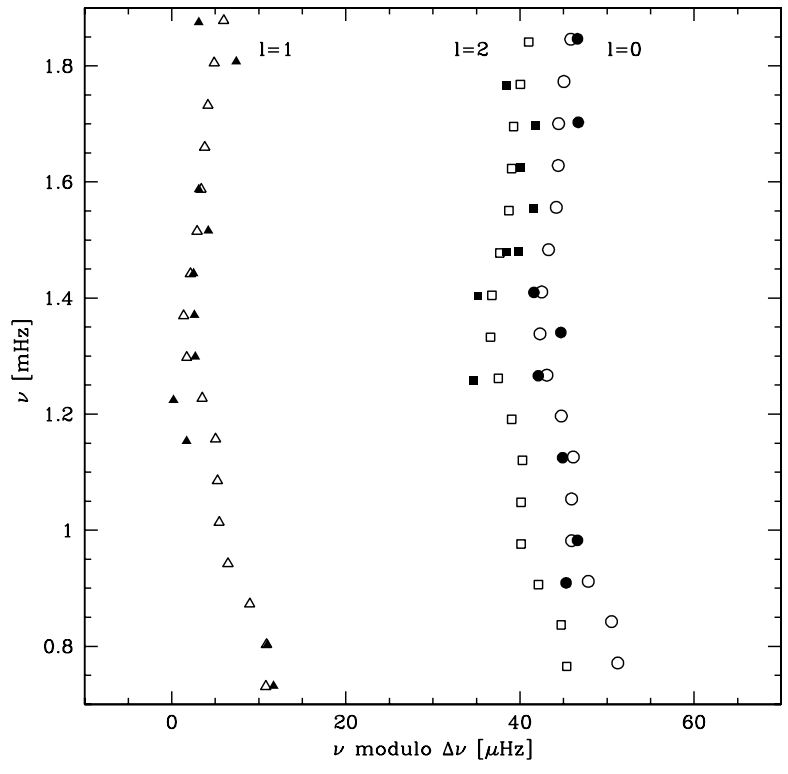

Fig. 6. Echelle diagram for the M 1 model. Open symbols refer to the theoretical frequencies, and filled symbols to the observed ones. Circles are used for $\ell=0$ modes, triangles for $\ell=1$ modes, and squares for $\ell=2$ modes. The two $\ell=2$ modes near $1480 \mu \mathrm{Hz}$ and separated by $1.4 \mu \mathrm{Hz}$ could be due to rotational splitting.

spacing near $1700 \mu \mathrm{Hz}$ than does the M 2 model, but the difference is clearly not significant.

Finally, theoretical and observed p-mode frequencies are compared by plotting the echelle diagram of the M 1 and M 2 models (Figs. 6 and 7). In these figures the systematic difference $\left\langle D_{v}\right\rangle$ between theoretical and observed frequencies has been taken into account $\left(\left\langle D_{v}\right\rangle=23.8 \mu \mathrm{Hz}\right.$ and $21.1 \mu \mathrm{Hz}$ for the M 1 and M 2 model, respectively). This shift of about $20 \mu \mathrm{Hz}$ between theoretical and observed frequencies is substantially larger than the shift required in the solar case. We recall that our models have been computed with the standard mixing-length formalism for convection and that we assume the oscillations to be adiabatic. It is beyond the scope of the present paper to investigate the influence of these simple assumptions on the solutions, but we note that they may explain a shift of about $20 \mu \mathrm{Hz}$ between computed and observed frequencies for a star that is more massive and more evolved than the Sun.

\section{Effects of rotation}

\subsection{Small spacings $\delta v_{02}$ and ratio $r_{02}$}

As already mentioned, the theoretical mean small spacings of the M 1 and M 2 models are smaller than the observed ones. The theoretical values are, however, perfectly compatible with the observations given the large uncertainty on the observed mean large spacing. Moreover, only three values of $\delta v_{02}$ (and two of $r_{02}$ ) have been observed. It is nevertheless interesting to investigate which physical process can increase the theoretical value of the mean small spacing in order to improve the agreement between the models and the seismological data.

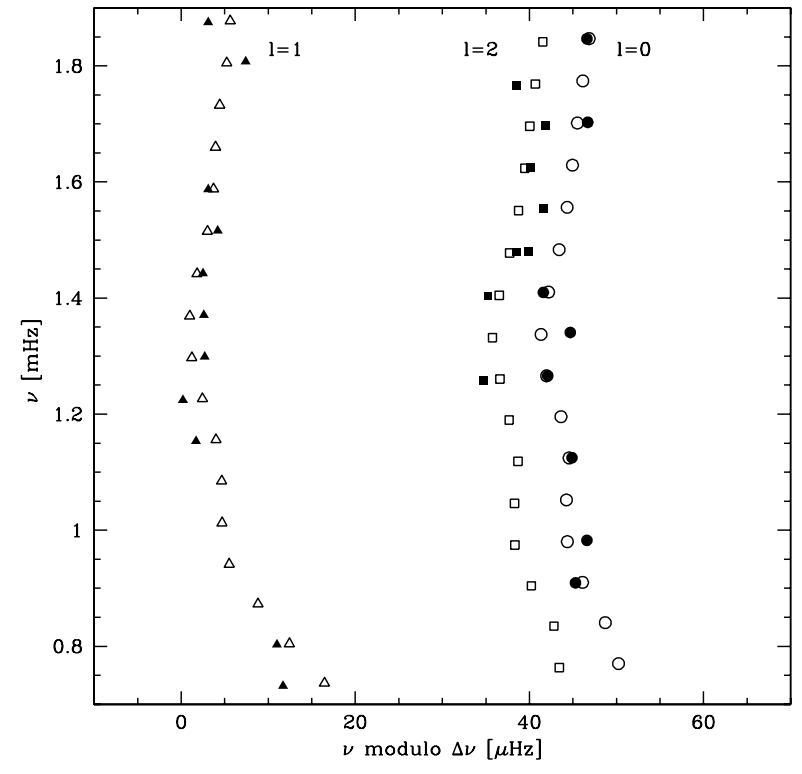

Fig. 7. Echelle diagram for the M 2 model. Open symbols refer to the theoretical frequencies, and filled symbols to the observed ones. Circles are used for $\ell=0$ modes, triangles for $\ell=1$ modes, and squares for $\ell=2$ modes.

Because the small spacing is principally sensitive to the structure of the core, its mean value decreases during the evolution of the star on the main sequence. Thus, younger models than the M 1 model, which is very near hydrogen exhaustion, exhibit higher values for the mean small spacing. Although these younger models are in better agreement with the observed values of $\delta v_{02}$, they fail to reproduce the other seismological data. Indeed, the mean large spacing also decreases during the evolution of the star on the main sequence due to the increase of its radius. Consequently, the mean large spacing of the younger models is larger than the observed one; these models are therefore unable to reproduce the observed individual frequencies.

It is thus necessary to find a process that is able to increase the mean small spacing without changing the mean large spacing. By changing the conditions in the central regions of the star, the rotational mixing is able to increase the values of $\delta v_{02}$ and $r_{02}$ for models with the same mean large spacing. To investigate the effects of rotational mixing on $\delta v_{02}$ and $r_{02}$, we compute models with exactly the same initial parameters as the M 1 model, but different initial velocities. The M 1 model is computed with an initial velocity of $18 \mathrm{~km} \mathrm{~s}^{-1}$ in order to reproduce the observed surface velocity of about $4 \mathrm{~km} \mathrm{~s}^{-1}$. We calculate two other models with higher initial rotation velocities of 50 and $100 \mathrm{~km} \mathrm{~s}^{-1}$. Both models are evolved until their mean large spacing equals the observed value of $72.1 \mu \mathrm{Hz}$. The surface velocity $V$ decreases during the evolution on the main sequence as a result of the magnetic braking that undergoes the low mass stars. When the observed value of the large spacing is reached, these velocities are equal to 6.8 and $8.2 \mathrm{~km} \mathrm{~s}^{-1}$ for the model with an initial velocity of 50 and $100 \mathrm{~km} \mathrm{~s}^{-1}$, respectively. 

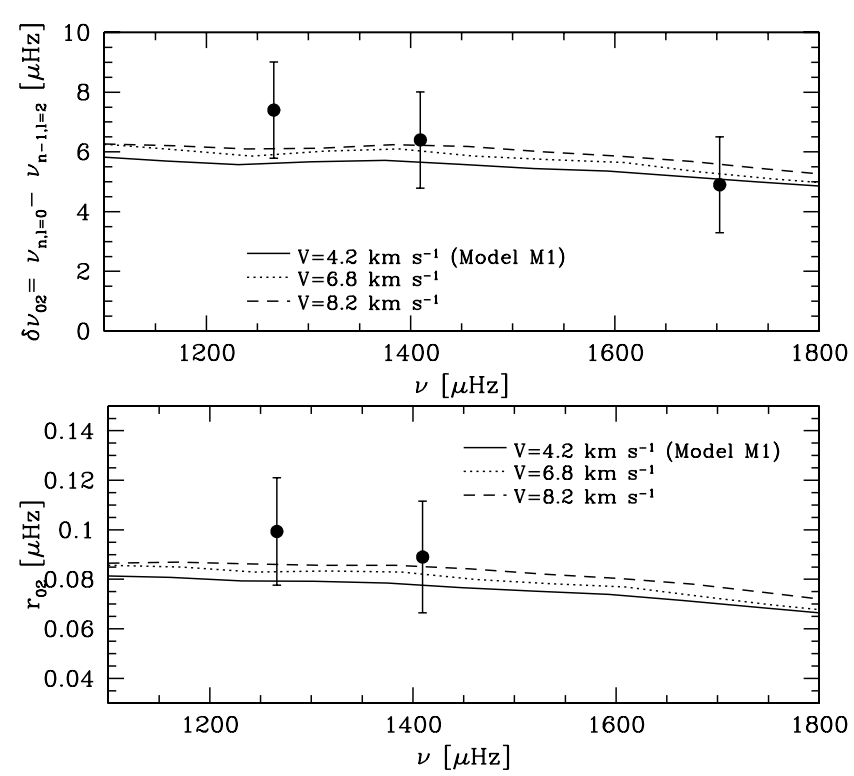

Fig. 8. Small spacing $\delta v_{02}$ and ratio of small to large separation $r_{02} \equiv$ $\delta v_{02} / \Delta v_{n, \ell=1}$ as a function of frequency for models with the same value of the mean large spacing $(72.1 \mu \mathrm{Hz})$ but different surface velocities $V$. Apart from the initial value of the rotational velocity, the models have been computed with the same initial parameters as the M 1 model. Dots indicate the observed values.

In this way, we obtain three models computed with the same input physics and initial parameters except for the initial velocity, and they are in good global agreement with the asteroseismic measurements. The models with a higher initial velocity are of course in poorer agreement with the classical constraints than the M 1 model, since an increase in the rotational velocity results in an increase in the effective temperature and luminosity. All models are, however, compatible with the adopted non-asteroseismic observational constraints. The variation in the small spacing $\delta v_{02}$ and the ratio $r_{02}$ with frequency for the three models is given in Fig. 8. This figure shows that an increase in the rotational velocity increases $\delta v_{02}$ and $r_{02}$. Indeed, the mean small spacing of the M 1 model is $5.4 \mu \mathrm{Hz}$, while it is equal to 5.7 and $6.0 \mu \mathrm{Hz}$ for the model with a surface velocity of 6.8 and $8.2 \mathrm{~km} \mathrm{~s}^{-1}$, respectively. This illustrates the fact that the mixing in the central layers, hence the values of $\delta v_{02}$ and $r_{02}$, increase with the rotational velocity.

\subsection{Surface abundances}

In addition to changing the structure of the stellar core, rotation also influences the chemical abundances at the surface of the star. Indeed, rotation-induced mixing counteracts the effects of atomic diffusion in the external layers of the star. Since the efficiency of this mixing increases with the rotational velocity, the decrease of the helium and heavy elements abundances due to atomic diffusion is found to be smaller for larger rotational velocities. This is illustrated in Fig. 9, which shows the helium profile in the external layers of the star for the three rotating models with the same mean large spacing of $72.1 \mu \mathrm{Hz}$. We see that the helium surface abundance is larger when the surface

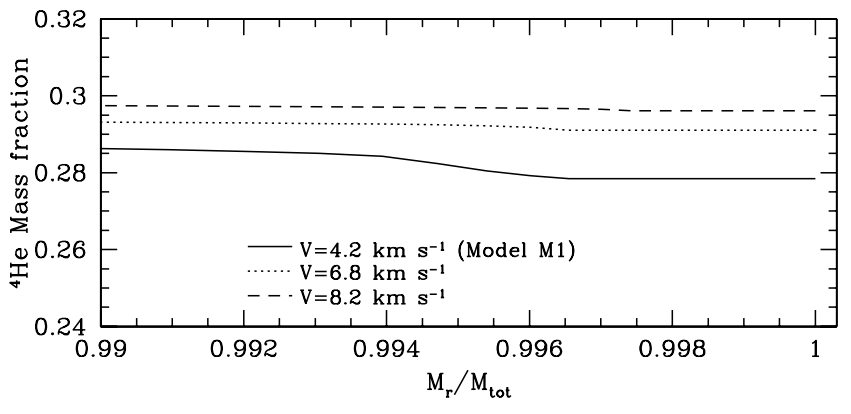

Fig. 9. Helium abundance profile in the external layers of the star for models with the same value of the mean large spacing $(72.1 \mu \mathrm{Hz})$ but different surface velocities $V$.
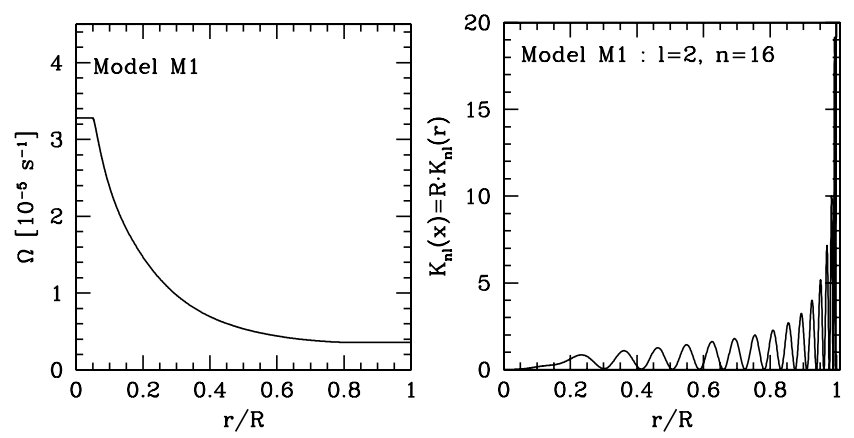

Fig. 10. Rotation profile of the M 1 model and the rotational kernel corresponding to the $\ell=2$ mode with $n=16$.

velocity is higher. Although the effects of rotation on the surface abundances are relatively small for stars with masses lower than $1.3 M_{\odot}$ like $\beta$ Vir, we note that they are particularly important for more massive stars with shallower convective envelope like Procyon or $\eta$ Bootis. Indeed, in stars that are more massive than about $1.4 M_{\odot}$, the rotation induced mixing prevents the helium and the heavy elements from being drained out of the convective envelope (see Fig. 10 of Carrier et al. 2005a for $\eta$ Bootis).

\subsection{Dispersion of the observed large spacings}

As mentioned above, the observed large spacings show a significant dispersion around the theoretical curves. Carrier et al. (2005b) suggest that this scatter can be partly explained by rotational splitting, since the dispersion for $\ell=2$ and $\ell=$ 1 modes are found to be greater than for radial modes. To check this, we compute the theoretical rotational splittings for the $\ell=2$ and $\ell=1$ modes of the M 1 model. These frequency splittings are calculated using

$\delta \omega_{n \ell m}=m \beta_{n \ell} \int_{0}^{R} K_{n \ell}(r) \Omega(r) \mathrm{d} r$,

where the rotational kernel $K_{n \ell}$ and the coefficients $\beta_{n \ell}$ are defined by Eqs. (8.42) and (8.43) from Christensen-Dalsgaard (2003). The rotation profile of the M 1 model and an example of the rotational kernel computed for the $\ell=2$ mode with $n=16$ are shown in Fig. 10. Using Eq. (10), 


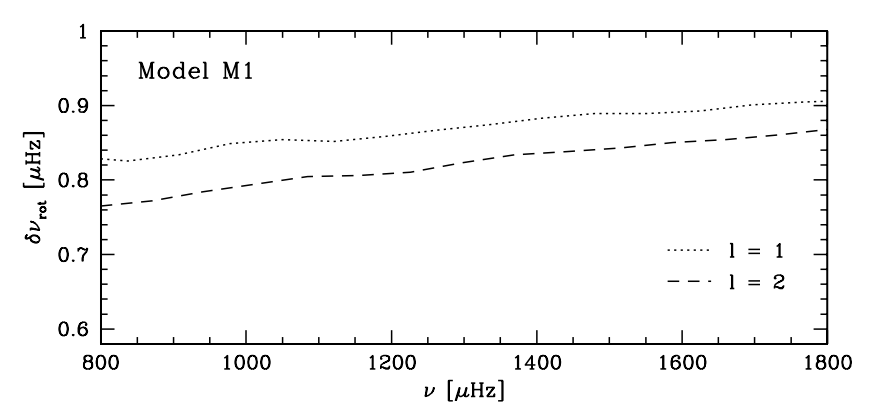

Fig. 11. Rotational splittings $\left(\delta v_{\mathrm{rot}}(n, \ell) \equiv \delta \omega_{n, \ell, m=1} / 2 \pi\right)$ for the M 1 model. The dotted and the dashed lines correspond to the splittings for modes with $\ell=1$ and $\ell=2$, respectively.

the rotational splittings $\delta v_{\text {rot }}$ expressed in terms of cyclic frequencies $\left(\delta v_{\mathrm{rot}}(n, \ell) \equiv \delta \omega_{n, \ell, m=1} / 2 \pi\right)$ are calculated for the $\ell=1$ and $\ell=2$ modes; they are shown in Fig. 11.

The theoretical rotational splittings lie between 0.8 and $0.9 \mu \mathrm{Hz}$ for $\ell=1$ modes, and between 0.75 and $0.85 \mu \mathrm{Hz}$ for $\ell=2$ modes. We notice that these values are higher than the value of about $0.6 \mu \mathrm{Hz}$ deduced by assuming a uniform rotation velocity equal to the angular velocity at the surface. This reflects the increase of the angular velocity $\Omega$ when the distance to the center of the star decreases (see Fig. 10). Due to the rotational splittings, the frequencies of the $\ell=1$ modes can be shifted by $\pm \delta v_{\text {rot }}(\ell=1)(\sim 0.85 \mu \mathrm{Hz})$, and the frequencies of the $\ell=2$ modes can be shifted by $\pm 2 \delta v_{\text {rot }}(\ell=2)(\sim 1.6 \mu \mathrm{Hz})$. This introduces a scatter of the frequencies which can explain the high dispersion of the observed large spacings. Figure 12 shows the theoretical dispersion of the large spacings expected from rotational splittings; the values for the $\ell=1$ modes are delimited by the modes with $m= \pm 1$, while the values for the $\ell=2$ modes are delimited by the modes with $m= \pm 2$.

We see that the scatter of frequencies resulting from the rotational splittings can account for the high dispersion of the observed large spacings and especially for the fact that this dispersion is greater for $\ell=2$ and $\ell=1$ modes than for radial modes. Indeed, the observed large spacings for the $\ell=1$ and $\ell=2$ modes are found to lie within the predicted interval in $\Delta v$. There is only one exception: the $\ell=1$ value near $1880 \mu \mathrm{Hz}$, which is too small to be correctly reproduced by the models. We also notice that the two values of the $\ell=0$ large spacing near $1400 \mu \mathrm{Hz}$ are not in good agreement with the theoretical predictions. This cannot, of course, been explained by rotational splittings, but can either be due to the scatter resulting from the finite lifetimes of the modes (which, contrary to the rotational splittings, affects all the modes including the radial ones) or/and to the misidentification of the observed frequency near $1340 \mu \mathrm{Hz}$. Indeed, the echelle diagrams show that the models fail to reproduce the $\ell=0$ frequency near $1340 \mu \mathrm{Hz}$ (see Figs. 6 and 7). As a result, the two observed large spacings for $\ell=0$ modes near $1400 \mu \mathrm{Hz}$ do not agree well with the theoretical ones. It is interesting to note that the extracted frequency corresponding to the $\ell=0$ frequency at $1340.7 \mu \mathrm{Hz}$ is $1329.1 \mu \mathrm{Hz}$ (see Table 2 in Carrier et al. 2005b). Thus, this frequency has been shifted by $+11.57 \mu \mathrm{Hz}$ by Carrier et al. and has
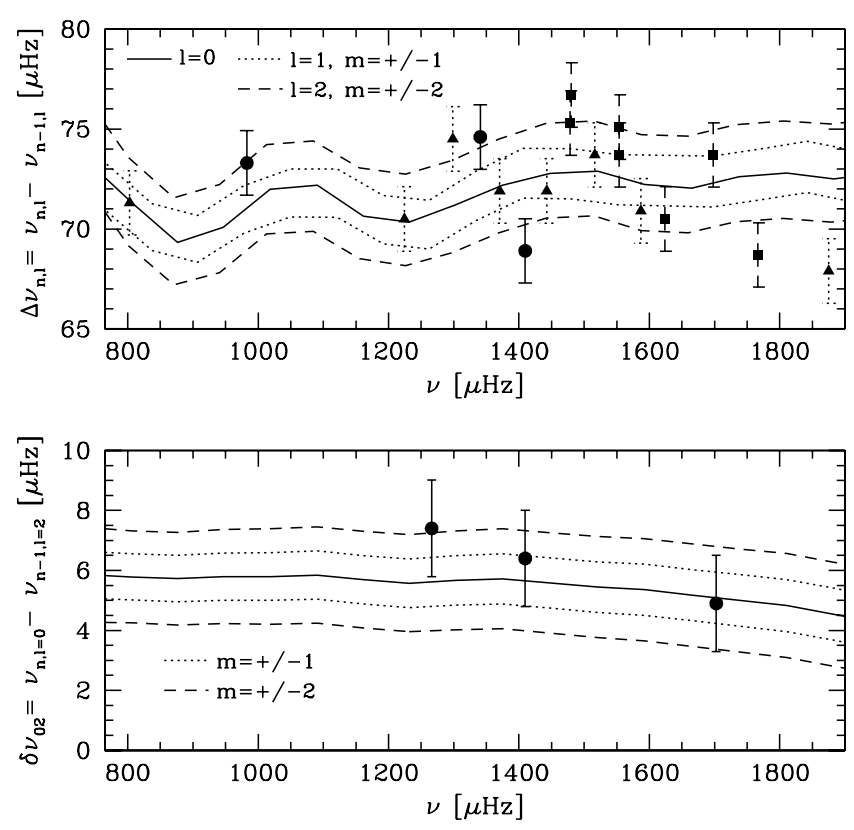

Fig. 12. Dispersion of the large and small spacings expected from rotational splittings for the M 1 model. The observed large spacings for the $\ell=1$ modes are expected to lie between the maximum/minimum values delimited by the $\ell=1, m= \pm 1$ modes. In the same way, the observed large spacings for the $\ell=2$ modes and the observed small spacings $\delta v_{02}$ are expected to lie between the maximum/minimum values delimited by the $\ell=2, m= \pm 2$ modes.

been identified as an $\ell=0$ mode. Comparison with our models shows that the extracted frequency of $1329.1 \mu \mathrm{Hz}$ in fact better agrees with the theoretical predictions than does the shifted frequency of $1340.7 \mu \mathrm{Hz}$. Thus, we think that this frequency, which was originally identified as a radial mode at $1340.7 \mu \mathrm{Hz}$ by Carrier et al., is in fact an $\ell=2$ mode at $1329.1 \mu \mathrm{Hz}$. The observed small spacings are also perfectly compatible with the theoretical predictions, when the uncertainties due to the rotational splittings of the $\ell=2$ modes are taken into account (see bottom of Fig. 12).

Figure 10 shows that models that include shellular rotation predict an increase in the angular velocity when the distance to the center decreases in contrast to the near uniformity of the solar rotation profile. Indeed, helioseismological results indicate that the angular velocity $\Omega(r)$ is constant as a function of the radius $r$ between about $20 \%$ and $70 \%$ of the total solar radius (Brown et al. 1989; Kosovichev et al. 1997; Couvidat et al. 2003). Since shellular rotation alone produces an insufficient internal coupling to ensure solid body rotation (Pinsonneault et al. 1989; Chaboyer et al. 1995; Eggenberger et al. 2005b), this suggests that another effect intervenes. Two mechanisms have been proposed as reproducing the solar rotation profile: magnetic fields (Mestel \& Weiss 1987; Charbonneau \& MacGregor 1993; Eggenberger et al. 2005b) and internal gravity waves (Zahn et al. 1997; Talon \& Charbonnel 2005). However, the question remains open as to how rotation should be modeled in a slightly more massive solar-type star like $\beta$ Virginis. It is therefore worth investigating how a flat 
rotation profile will affect our conclusions about the effects of rotation on the small separations and the rotational splittings of $\beta$ Virginis. The near solid body rotation of models including magnetics fields results in a low value for the diffusion coefficient associated to the shear turbulent mixing, and suggests that, for slow rotating solar-like stars, rotational mixing is less efficient in magnetic models than in models with only rotation (Eggenberger et al. 2005b). In the same way, the inclusion of internal gravity waves is found to reduce rotational mixing together with differential rotation (Talon \& Charbonnel 2005). The mixing in the central layers for a model of $\beta$ Virginis exhibiting a solar-like rotation profile will thus be reduced resulting in a less pronounced increase in $\delta v_{02}$ and $r_{02}$ with the rotational velocity. As mentioned above, the values of the rotational splittings will also be influenced: the rotational splittings computed for a model of $\beta$ Virginis with a uniform rotation velocity are smaller than the splittings corresponding to the rotation profile of our M 1 model (mean value of $0.57 \mu \mathrm{Hz}$ instead of $0.83 \mu \mathrm{Hz}$ ). This results in a smaller scatter in the frequencies of non-radial modes for models with a solar-like rotation profile than for our M 1 model. It is, however, clear that the accuracy of the present asteroseismic observations does not enable us to distinguish between these models with different rotation profiles. We can simply hope that future asteroseismic observations will lead to accurate determination of rotational splittings and small separations for stars with various masses and ages, in order to provide us with new insight into the rotation of solartype stars and with constraints on how to model it best.

\section{Conclusion}

The aim of this work was to determine the best model for the F9 V star $\beta$ Virginis using the Geneva evolution code. By combining the existing non-asteroseismic observables with the new seismological measurements by Carrier et al. (2005b), we find that two different solutions reproduce all these constraints well: a main-sequence model with a mass of $1.28 \pm$ $0.03 M_{\odot}$, an age $t=3.24 \pm 0.20 \mathrm{Gyr}$, and an initial metallicity $(Z / X)_{\mathrm{i}}=0.0340 \pm 0.0040$, or else a model in the postmain sequence phase of evolution with a lower mass of $1.21 \pm$ $0.02 M_{\odot}$ and a larger age $t=4.01 \pm 0.30 \mathrm{Gyr}$. Although these two models are compatible with all observables, we note that the main-sequence model agrees slightly better with the nonasteroseismic observables than the post-main-sequence one.

We show that the small spacings $\delta v_{02}$ and the ratio $r_{02}$ between small and large spacings are sensitive to the differences in the structure of the central layers between the mainsequence and the post-main-sequence model; therefore, they can be used to unambiguously determine the evolutionary stage of $\beta$ Vir. Unfortunately, existing asteroseismic data do not enable such a determination, since none of the observed $\delta v_{02}$ and $r_{02}$ are available for frequencies where significant differences between the values of $\delta v_{02}$ and $r_{02}$ for main-sequence and post-main-sequence models are predicted.

The effects of rotation were also studied. We first find that the changes in the inner structure of the star due to the rotational mixing may be revealed by asteroseismic observations, if precise measurements of $\delta v_{02}$ and $r_{02}$ can be made. We also show that the scatter of frequencies introduced by the rotational splittings can account for the larger dispersion of the observed large spacings for the non-radial modes than for the radial modes

We conclude that the combination of non-asteroseismic measurements with existing frequency observations puts important constraints on the global parameters of $\beta$ Vir, but that additional asteroseismic measurements are needed (especially measurements of the small spacings) to unambiguously determine its evolutionary stage and to investigate the internal structure of this star in detail.

Acknowledgements. We thank J. Christensen-Dalsgaard for providing us with the Aarhus adiabatic pulsation code. We also thank A. Maeder, G. Meynet, C. Charbonnel, and S. Talon for helpful advice. This work was supported financially by the Swiss National Science Foundation.

\section{References}

Allende Prieto, C., Barklem, P. S., Lambert, D. L., \& Cunha, K. 2004, A\&A, 420, 183

Angulo, C., Arnould, M., Rayet, M., et al. 1999, Nucl. Phys. A, 656, 3

Bedding, T. R., Kjeldsen, H., Butler, R. P., et al. 2004, ApJ, 614, 380

Bouchy, F., \& Carrier, F. 2002, A\&A, 390, 205

Bouchy, F., Bazot, M., Santos, N. C., et al. 2005, A\&A, 440, 609

Bouvier, J., Forestini, M., \& Allain, S. 1997, A\&A, 326, 1023

Brown, J. M., Christensen-Dalsgaard, J., Dziembowski, W. A., et al. 1989, ApJ, 343, 526

Burki, G., et al. 2005, http://obswww. unige.ch/gcpd/ph13.html

Buzzoni, A., Chavez, M., Malagnini, M. L., \& Morossi, C. 2001, PASP, 113, 1365

Carrier, F., \& Bourban, G. 2003, A\&A, 406, L23

Carrier, F., Eggenberger, P., \& Bouchy, F. 2005a, A\&A, 434, 1085

Carrier, F., Eggenberger, P., D’Alessandro, A., \& Weber, L. 2005b, New Astron., 10, 315

Chaboyer, B., \& Zahn, J. P. 1992, A\&A, 253, 173

Chaboyer, B., Demarque, P., \& Pinsonneault, M. H. 1995, ApJ, 441, 865

Charbonneau, P., \& MacGregor, K. B. 1993, ApJ, 417, 762

Christensen-Dalsgaard, J. 1997, http://astro.phys.au.dk/ jcd/adipack.n/

Christensen-Dalsgaard, J. 2003, Lecture Notes on Stellar Oscillations, 5 th Ed.

Christensen-Dalsgaard, J., Däppen, W., \& Lebreton, Y. 1988, Nature, 336,634

Couvidat, S., García, R. A., Turck-Chièze, S., et al. 2003, ApJ, 597, $77 \mathrm{~L}$

Di Mauro, M. P., Christensen-Dalsgaard, J., Kjeldsen, H., et al. 2003, A\&A, 404, 341

Edvardsson, B., Andersen, J., Gustafsson, B., et al. 1993, A\&A, 275, 101

Eggenberger, P., Carrier, F., Bouchy, F., \& Blecha, A. 2004a, A\&A, 422,247

Eggenberger, P., Charbonnel, C., Talon, S., et al. 2004b, A\&A, 417, 235

Eggenberger, P., Carrier, F., \& Bouchy, F. 2005a, New Astron., 10, 195

Eggenberger, P., Maeder, A., \& Meynet, G. 2005b, A\&A, 440, L9

Frandsen, S., Carrier, F., Aerts, C., et al. 2002, A\&A, 394, L5

Flower, P. 1996, ApJ, 469, 355

Glebocki, R., \& Stawikowski, A. 2000, AcA, 50, 509 
Gray, R. O., Graham, P. W., \& Hoyt, S. R. 2001, AJ, 121, 2159

Grevesse, N., \& Sauval, A. J. 1998, Space Sci. Rev., 85, 161

Guenther, D. B. 1994, ApJ, 422, 400

Guenther, D. B., \& Brown, K. I. T. 2004, ApJ, 600, 419

Kawaler, S. D. 1988, ApJ, 333, 236

Kjeldsen, H., Bedding, T. R., Baldry, I. K., et al. 2003, AJ, 126, 1483

Kjeldsen, H., Bedding, T. R., Butler, R. P., et al. 2005, ApJ, 635, 1281

Kosovichev, A., Schou, J., Scherrer, P. H., et al. 1997, Sol. Phys., 170, 43

Lambert, D. L., \& Reddy, B. E. 2004, MNRAS, 349, 757

Le Borgne, J.-F., Bruzual, G., Pelló, R., et al. 2003, A\&A, 402, 433

Lejeune, T., Cuisinier, F., \& Buser, R. 1998, A\&AS, 130, 65

Li, L. H., Robinson, F. J., Demarque, P., et al. 2002, ApJ, 567, 1192

Maeder, A. 2003, A\&A, 399, 263

Maeder, A., \& Zahn, J. P. 1998, A\&A, 334, 1000

Maeder, A., \& Meynet, G. 2001, A\&A, 373, 555

Martic, M., Lebrun, J.-C., Appourchaux, T., \& Korzennik, S. G. 2004a, A\&A, 418, 295
Martic, M., Lebrun, J.-C., Appourchaux, T., \& Schmitt, J. 2004b, in Helio- and Asteroseismology: Towards a Golden Future, Proc. SOHO 14/GONG 2004 Workshop, ESA SP-559, 563

Mestel, L., \& Weiss, N. O. 1987, MNRAS, 226, 123

Meynet, G., \& Maeder, A. 2000, A\&A, 361, 101

Morel, T., \& Micela, G. 2004, A\&A, 423, 677

Otí Floranes, H., Christensen-Dalsgaard, J., \& Thompson, M. J. 2004, MNRAS, 356, 671

Paquette, C., Pelletier, C., Fontaine, G., \& Michaud, G. 1986, ApJ, 61, 177

Pinsonneault, M. H., Kawaler, S. D., Sofia, S., \& Demarque, P. 1989, ApJ, 338, 424

Roxburgh, I. W., \& Vorontsov, S. V. 2003, A\&A, 411, 215

Taylor, A. R., Gibson, S. J., Peracaula, M., et al. 2003, AJ, 125, 3145

Talon, S., \& Charbonnel, C. 2005, A\&A, 440, 981

Ulrich, R. K., \& Rhodes, E. J. Jr. 1983, ApJ, 265, 551

Zahn, J. P. 1992, A\&A, 265, 115

Zahn, J. P., Talon, S., \& Matias, J. 1997, A\&A, 322, 320 